\title{
Suzuki coupling of aryl chlorides with phenylboronic acid in water using microwave heating with simultaneous cooling
}

Riina K. Arvela and Nicholas. E. Leadbeater

Department of Chemistry, University of Connecticut, Unit 3060, 55 North Eagleville Road, Storrs, CT 06269-3060, USA

nicholas.leadbeater@uconn.edu

\section{Supporting Information}

General experimental $\quad$ S2

Typical experimental procedure $\quad$ S3

${ }^{1} \mathrm{H}$ and ${ }^{13} \mathrm{C}$ NMR for the compounds prepared S4-S23 


\section{General experimental}

Unless noted otherwise, all materials were obtained from commercial suppliers and used without further purification. 10\% Palladium on carbon was purchased from Aldrich. All reactions were carried out in air. Reactions were conducted using a focused microwave unit. The machine consists of a continuous focused microwave power delivery system with operator selectable power output from 0-300 W. Reactions were performed in glass vessels (capacity $10 \mathrm{~mL}$ ) sealed with a pressure lock. The pressure is controlled by a load cell connected directly to the vessel. The temperature of the contents of the vessel was monitored using a calibrated fiber-optic device inserted in to the reaction vessel to a level $1.5 \mathrm{~cm}$ from the bottom of the tube. ${ }^{1} \mathrm{H}-$ and ${ }^{13} \mathrm{C}-\mathrm{NMR}$ spectra were recorded at $293 \mathrm{~K}$ on a $500 \mathrm{MHz}$ spectrometer. 


\section{Typical experimental procedure}

In a $10 \mathrm{~mL}$ glass tube was placed aryl chloride $(1.0 \mathrm{mmol})$, phenylboronic acid $(0.159 \mathrm{~g}$, $1.3 \mathrm{mmol}$ ), $\mathrm{Na}_{2} \mathrm{CO}_{3}(392 \mathrm{mg}, 3.7 \mathrm{mmol})$, tetrabutylammonium bromide (322 mg, 1.0 $\mathrm{mmol}), 10 \% \mathrm{Pd} / \mathrm{C}(10.6 \mathrm{mg}, 0.01 \mathrm{~mol} \mathrm{Pd})$ water $(2 \mathrm{~mL})$ and magnetic stir bar. The vessel was placed into the microwave cavity and sealed with a pressure lock. A fiber optic temperature measurement device was inserted to reaction vessel through the pressure lock. Before the reaction were run, the mixture was stirred for 20 seconds to ensure sufficient mixing of the reagents. The microwave source was then turned on and microwave irradiation of $300 \mathrm{~W}$ was used to ramp the temperature from r.t. to $120{ }^{\circ} \mathrm{C}$ where it was held by modulating the microwave power until a total reaction time of 10 minutes had elapsed. After allowing the reaction mixture to cool down to r.t., the vessel was opened and the contents poured into a separatory funnel. Water $(30 \mathrm{~mL})$ and ethyl acetate $(30 \mathrm{~mL})$ were added and the organic material extracted and removed. After further extraction of the aqueous layer with ethyl acetate, combining the organic washings and drying them over $\mathrm{MgSO}_{4}$, the solvent was removed in vacuuo leaving the crude product. Products were purified and isolated by chromatography using hexane / ethyl acetate as eluent. 


\section{Spectroscopic data}

4-Methylbiphenyl (compound 1): ${ }^{1} \mathrm{H}$ NMR $\left(500 \mathrm{MHz}, \mathrm{CDCl}_{3}\right) \delta 7.64(\mathrm{~d}, \mathrm{~J}=7.1 \mathrm{~Hz}, 2 \mathrm{H})$, $7.55(\mathrm{~d}, \mathrm{~J}=8.1 \mathrm{~Hz}, 2 \mathrm{H}), 7.48(\mathrm{t}, \mathrm{J}=7.5 \mathrm{~Hz}, 2 \mathrm{H}), 7.37(\mathrm{t}, \mathrm{J}=7.3 \mathrm{~Hz}, 1 \mathrm{H}), 7.30$ (d, J = 7.9 Hz, 2H), 2.45 (s, 3H) ); ${ }^{13} \mathrm{C}$ NMR (500 MHz, $\left.\mathrm{CDCl}_{3}\right) \delta 141.2,138.4,137.0,129.5,128.7,127.2$, 127.0, 21.1.

4-Acetylbiphenyl (compound 2): ${ }^{1} \mathrm{H}$ NMR (500 MHz, $\left.\mathrm{CDCl}_{3}\right) \delta 8.06(\mathrm{~d}, \mathrm{~J}=8.2 \mathrm{~Hz}, 2 \mathrm{H})$, $7.72(\mathrm{~d}, \mathrm{~J}=8.2 \mathrm{~Hz}, 2 \mathrm{H}), 7.66(\mathrm{~d}, \mathrm{~J}=7.7 \mathrm{~Hz}, 2 \mathrm{H}), 7.50(\mathrm{t}, \mathrm{J}=7.5 \mathrm{~Hz}, 2 \mathrm{H}), 7.43(\mathrm{t}, \mathrm{J}=7.3 \mathrm{~Hz}$, 1H), $2.67(\mathrm{~s}, 3 \mathrm{H}) ;{ }^{13} \mathrm{C} \mathrm{NMR}\left(500 \mathrm{MHz}, \mathrm{CDCl}_{3}\right) \delta 197.8,145.8,139.9,135.9,129.0,128.9$, $128.3,127.3,127.2,26.7$.

4-Nitrobiphenyl (compound 3): ${ }^{1} \mathrm{H}$ NMR (500 MHz, $\left.\mathrm{CDCl}_{3}\right) \delta 8.32(\mathrm{~d}, \mathrm{~J}=8.4 \mathrm{~Hz}, 2 \mathrm{H})$, $7.76(\mathrm{~d}, \mathrm{~J}=8.4 \mathrm{~Hz}, 2 \mathrm{H}), 7.66(\mathrm{~d}, \mathrm{~J}=7.5 \mathrm{~Hz}, 2 \mathrm{H}), 7.53(\mathrm{t}, \mathrm{J}=7.4 \mathrm{~Hz}, 2 \mathrm{H}), 7.46-7.49(\mathrm{~m}, 1 \mathrm{H})$; ${ }^{13} \mathrm{C} \mathrm{NMR}\left(500 \mathrm{MHz}, \mathrm{CDCl}_{3}\right) \delta$ 147.6, 147.1, 138.8, 129.2, 129.0, 127.8, 127.4, 124.1.

4-Cyanobiphenyl (compound 4): ${ }^{1} \mathrm{H}$ NMR $\left(500 \mathrm{MHz}, \mathrm{CDCl}_{3}\right) \delta 7.75(\mathrm{~d}, \mathrm{~J}=8.5 \mathrm{~Hz}, 2 \mathrm{H})$, $7.71(\mathrm{~d}, \mathrm{~J}=8.5 \mathrm{~Hz}, 2 \mathrm{H}), 7.62(\mathrm{~d}, \mathrm{~J}=7.7 \mathrm{~Hz}, 2 \mathrm{H}), 7.52(\mathrm{t}, \mathrm{J}=7.5 \mathrm{~Hz}, 2 \mathrm{H}), 7.46(\mathrm{t}, \mathrm{J}=7.4 \mathrm{~Hz}$, 1H); ${ }^{13} \mathrm{C}$ NMR (500 MHz, $\left.\mathrm{CDCl}_{3}\right) \delta 145.7,139.2,132.6,129.1,128.7,127.8,127.3,119.0$, 110.9 .

2-Methylbiphenyl (compound 5): ${ }^{1} \mathrm{H} \mathrm{NMR}\left(500 \mathrm{MHz}, \mathrm{CDCl}_{3}\right) \delta 7.45(\mathrm{t}, \mathrm{J}=7.3 \mathrm{~Hz}, 2 \mathrm{H})$, 7.35-7.39 (m, 3H), 7.27-7.30 (m, 4H), $2.31(\mathrm{~s}, 3 \mathrm{H}) ;{ }^{13} \mathrm{C} \mathrm{NMR}\left(500 \mathrm{MHz}, \mathrm{CDCl}_{3}\right) \delta$ 142.0, $135.4,130.3,129.8,129.2,128.0,127.3,126.8,125.8,20.5$. 
Biphenyl (compound 6): ${ }^{1} \mathrm{H}$ NMR $\left(500 \mathrm{MHz}, \mathrm{CDCl}_{3}\right) \delta 7.64(\mathrm{dd}, \mathrm{J}=8.5 \mathrm{~Hz}, \mathrm{~J}=1.2 \mathrm{~Hz}$, $4 \mathrm{H}), 7.48(\mathrm{t}, \mathrm{J}=7.7 \mathrm{~Hz}, 4 \mathrm{H}), 7.39(\mathrm{tt}, \mathrm{J}=7.4 \mathrm{~Hz}, \mathrm{~J}=1.4 \mathrm{~Hz}, 2 \mathrm{H}) ;{ }^{13} \mathrm{C}$ NMR $(500 \mathrm{MHz}$, $\left.\mathrm{CDCl}_{3}\right) \delta 141.3,128.8,127.3,127.2$.

4-Methoxybiphenyl (compound 7): ${ }^{1} \mathrm{H}$ NMR (500 MHz, $\left.\mathrm{CDCl}_{3}\right) \delta$ 7.53-7.58 (m, 4H), 7.43 $(\mathrm{t}, \mathrm{J}=7.7 \mathrm{~Hz}, 2 \mathrm{H}), 7.32(\mathrm{t}, \mathrm{J}=7.4 \mathrm{~Hz}, 1 \mathrm{H}), 6.99(\mathrm{~d}, \mathrm{~J}=8.8 \mathrm{~Hz}, 2 \mathrm{H}), 3.86(\mathrm{~s}, 3 \mathrm{H}) ;{ }^{13} \mathrm{C} \mathrm{NMR}$ (500 MHz, $\left.\mathrm{CDCl}_{3}\right) \delta 159.2,140.9,133.8,128.7,128.2,126.8,126.7,114.2,55.4$.

2-Methoxybiphenyl (compound 8): ${ }^{1} \mathrm{H}$ NMR $\left(500 \mathrm{MHz}, \mathrm{CDCl}_{3}\right) \delta 7.60(\mathrm{~d}, \mathrm{~J}=7.7 \mathrm{~Hz}$, 2H), $7.47(\mathrm{t}, \mathrm{J}=7.6 \mathrm{~Hz}, 2 \mathrm{H})$ 7.37-7.40 (m, 3H), $7.10(\mathrm{~d}, \mathrm{~J}=7.5 \mathrm{~Hz}, 1 \mathrm{H}), 7.04(\mathrm{~d}, \mathrm{~J}=8.6 \mathrm{~Hz}$, 1H), 3.87 (s, 3H); ${ }^{13} \mathrm{C}$ NMR (500 MHz, $\left.\mathrm{CDCl}_{3}\right) \delta$ 156.5, 138.6, 130.9, 130.8, 129.6, 128.7, $128.0,127.0,120.9,111.3,55.6$.

4-Phenylaniline (compound 9): ${ }^{1} \mathrm{H}$ NMR $\left(500 \mathrm{MHz}, \mathrm{CDCl}_{3}\right) \delta 7.56(\mathrm{~d}, \mathrm{~J}=7.4 \mathrm{~Hz}, 2 \mathrm{H})$, 7.40-7.45 (m, 4H), 7.28-7.31 (m, 1H), $6.79(\mathrm{dd}, \mathrm{J}=8.2 \mathrm{~Hz}, \mathrm{~J}=1.6 \mathrm{~Hz}, 2 \mathrm{H}), 3.7(\mathrm{bs}, 2 \mathrm{H}) ;{ }^{13} \mathrm{C}$ NMR (500 MHz, $\left.\mathrm{CDCl}_{3}\right) \delta$ 145.8, 141.2, 131.6, 128.7, 128.0, 126.4, 126.3, 115.4. 
4-Methylbiphenyl

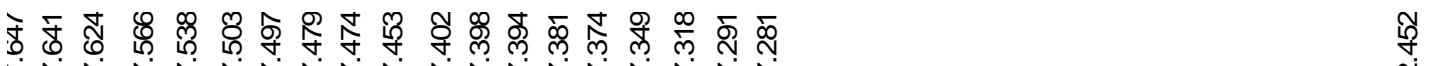
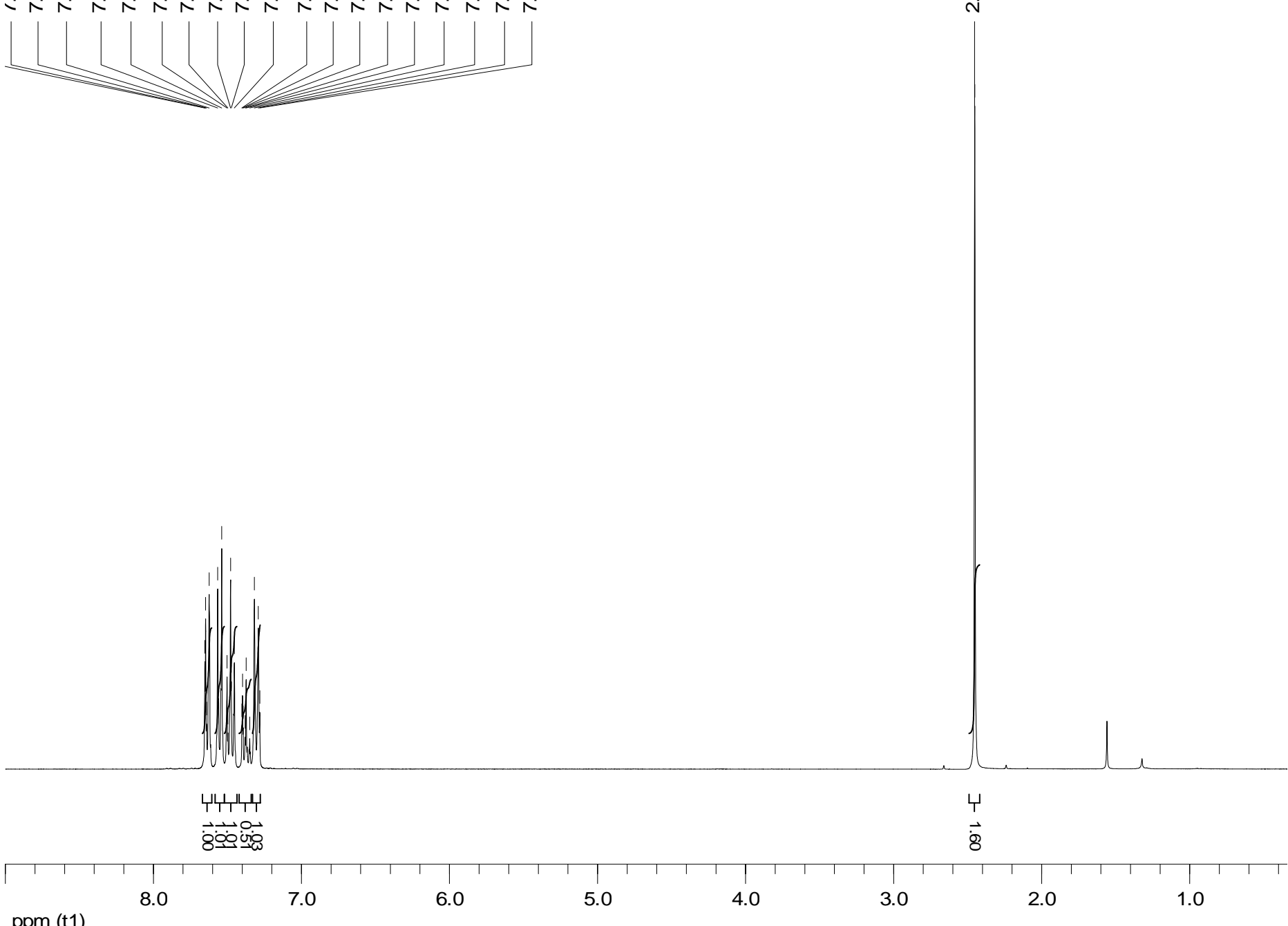

ppm (t1) 
4-Methylbiphenyl

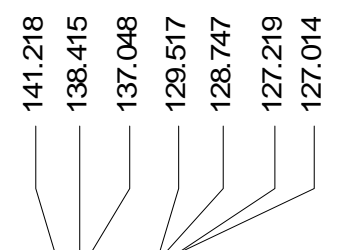

品 范

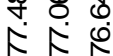

$\stackrel{\widehat{m}}{\vec{N}}$

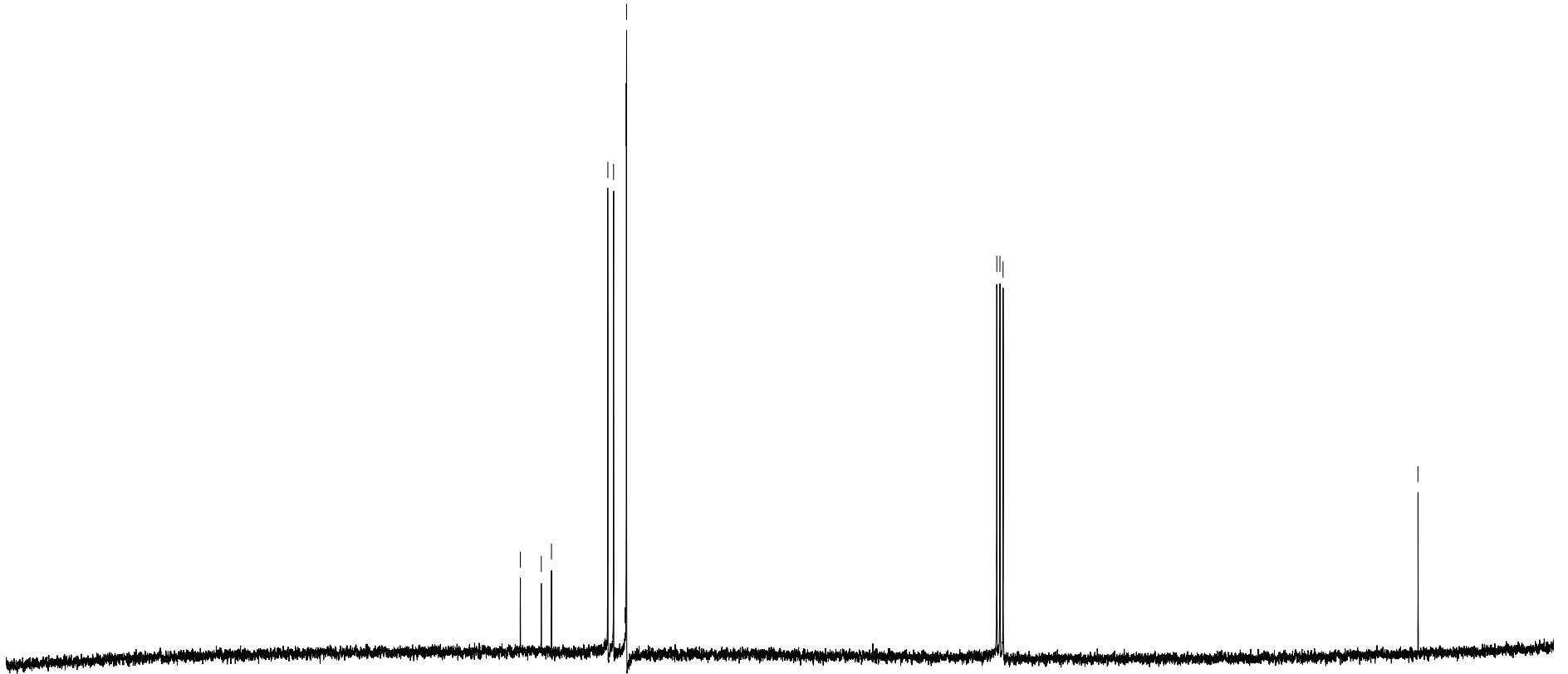


4-Acetylbiphenyl

空员
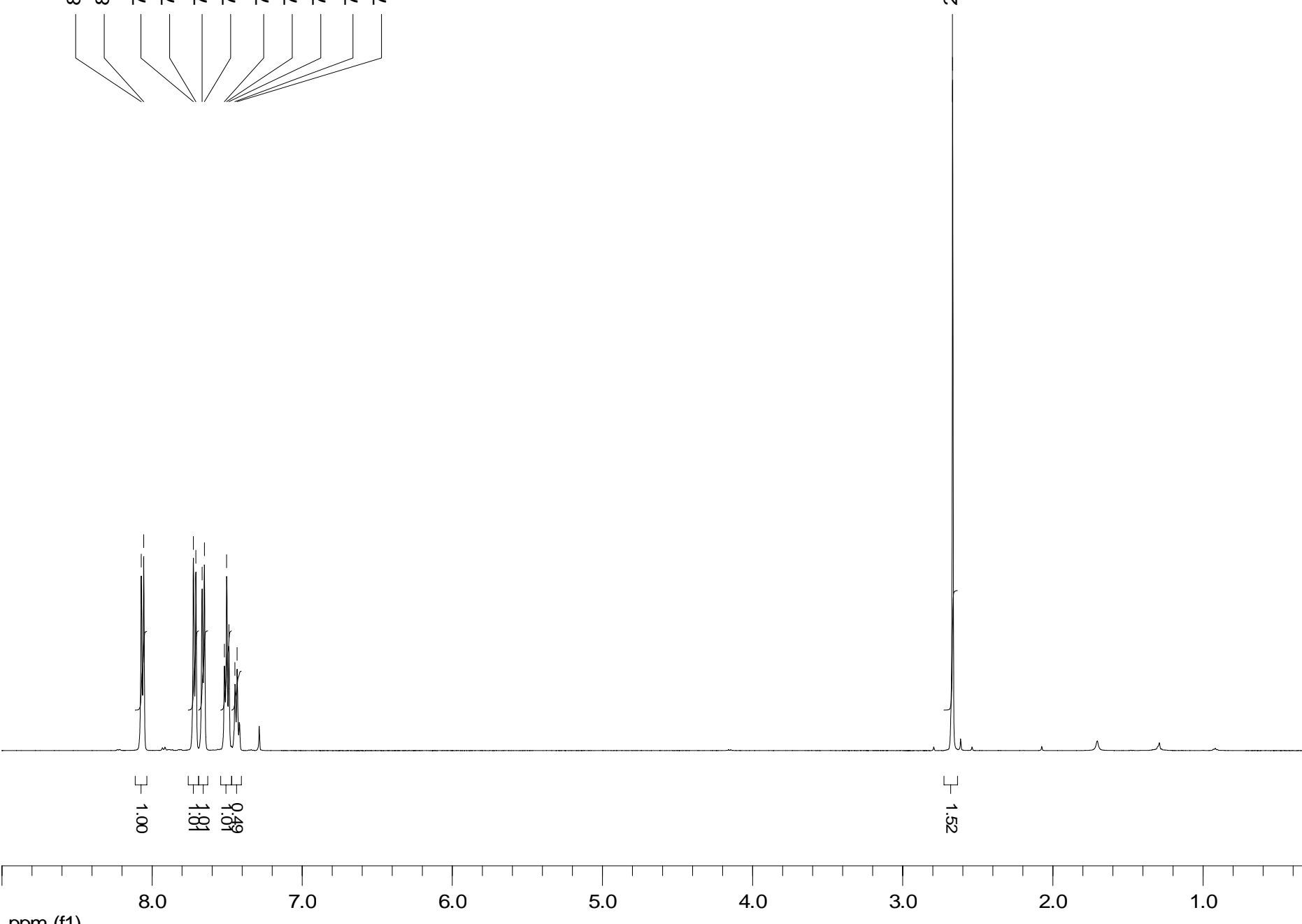

ppm (f1) 
4-Acetylbiphenyl
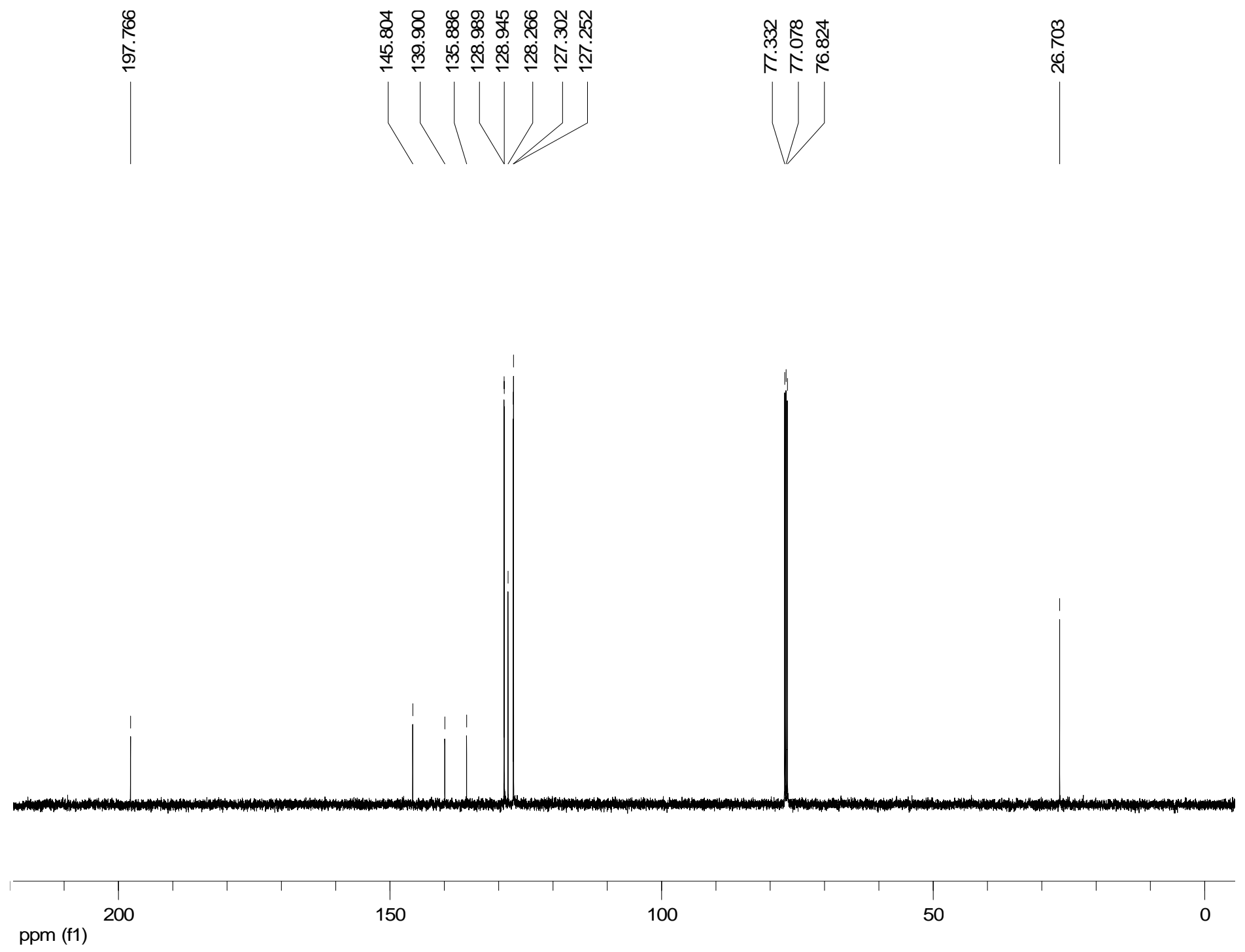


\section{4-Nitrobiphenyl}

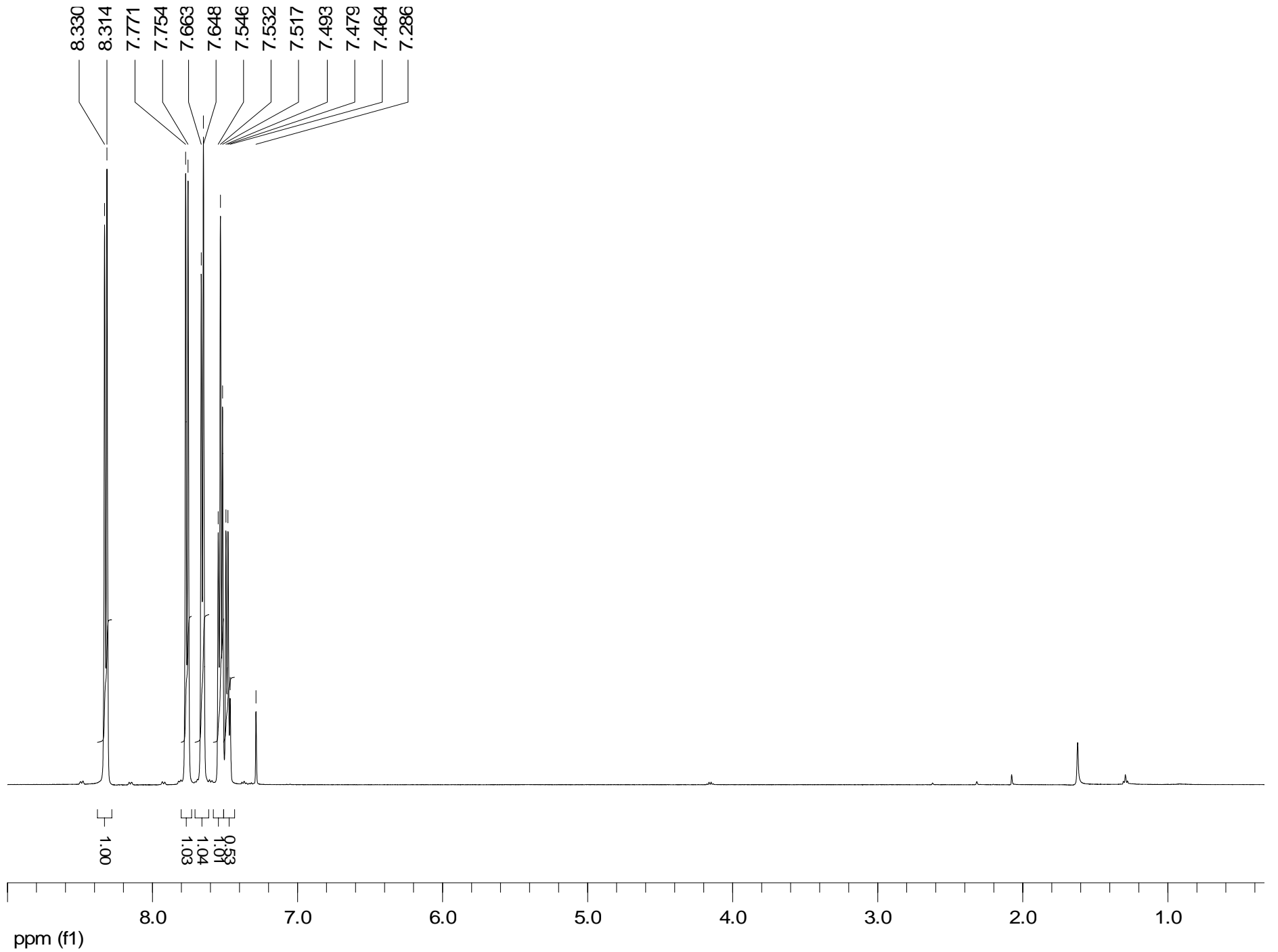


4-Nitrobiphenyl

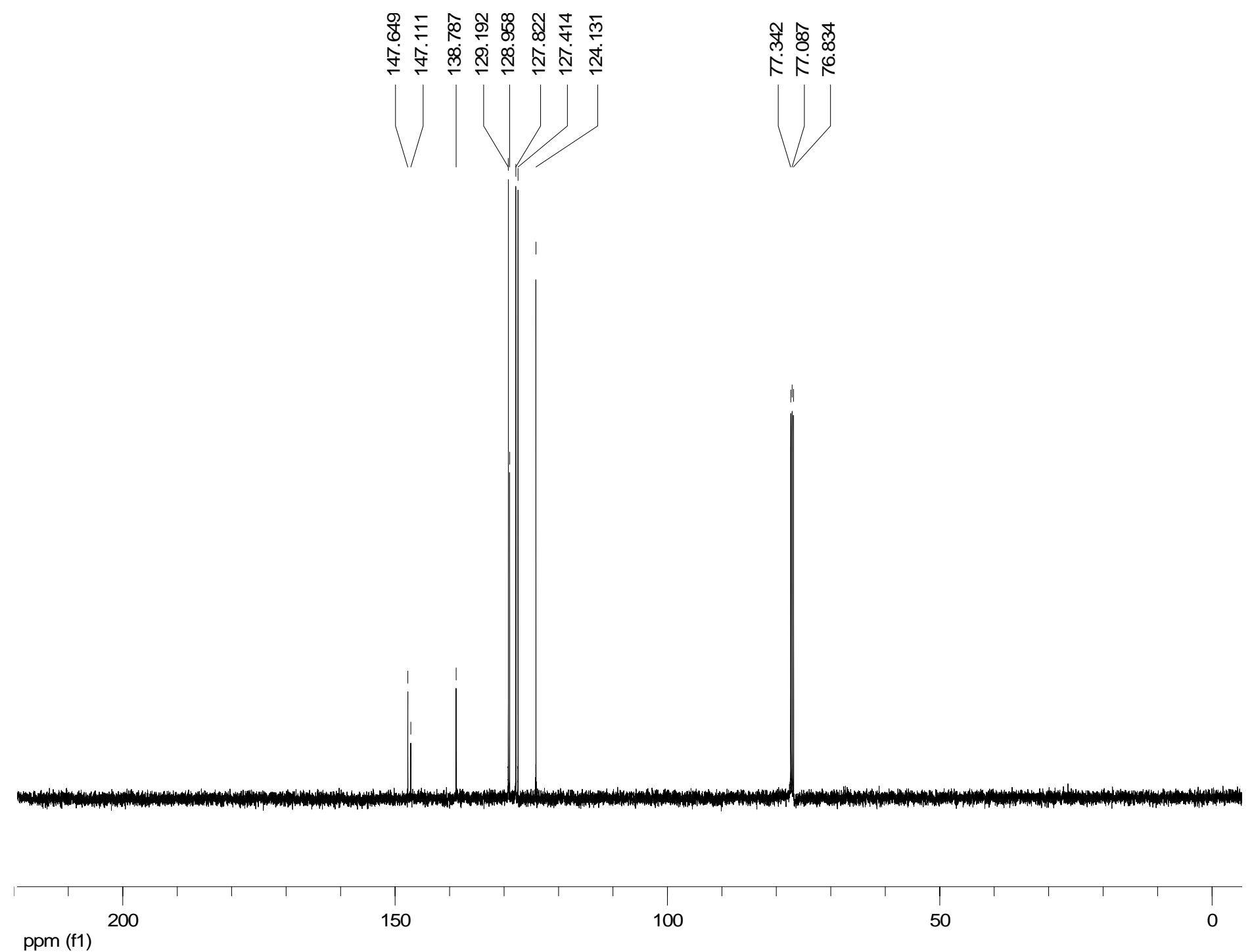

ppm (f1)

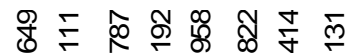

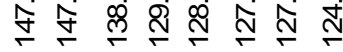

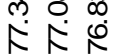


4-Cyanobiphenyl

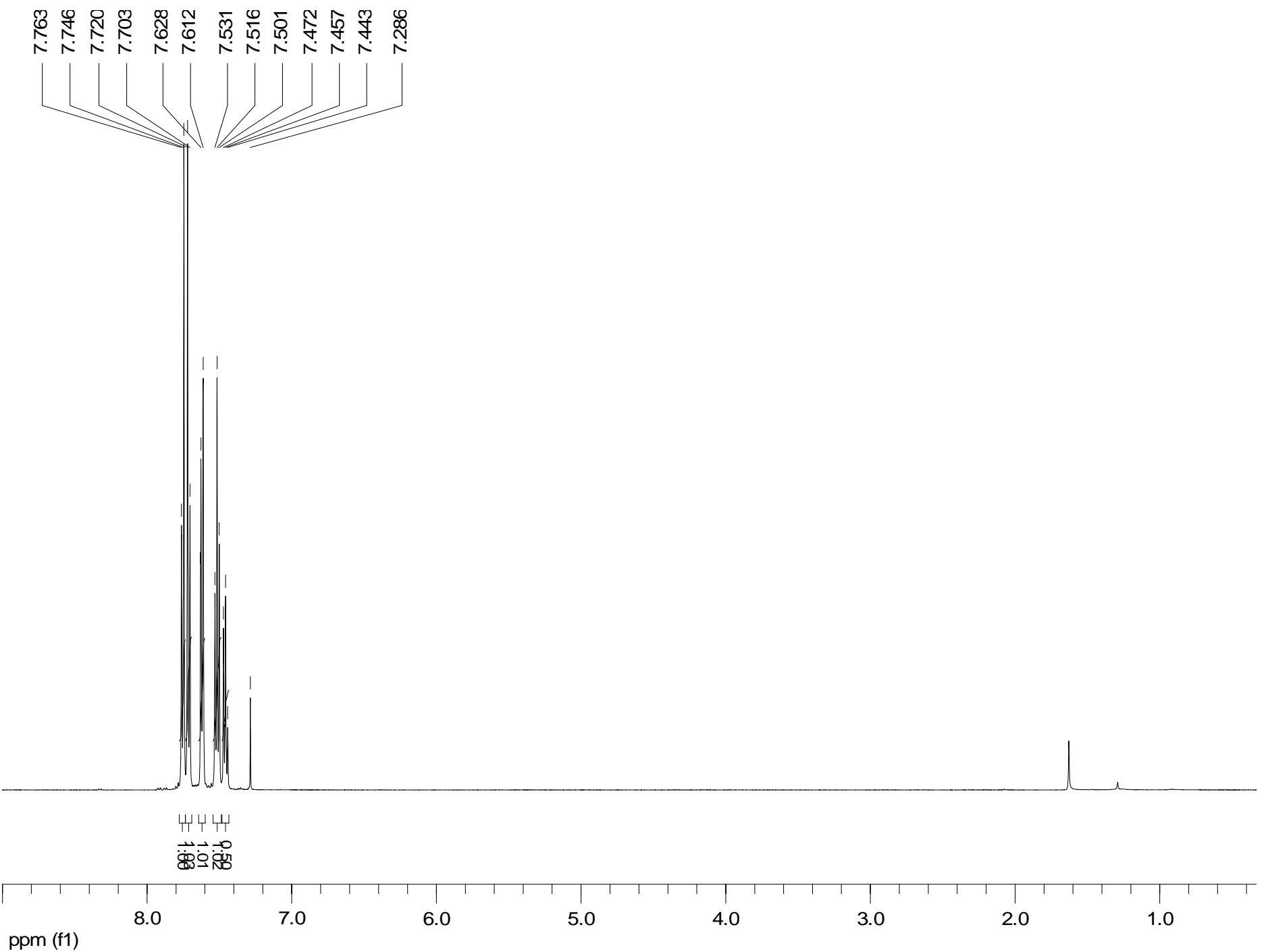


4-Cyanobiphenyl

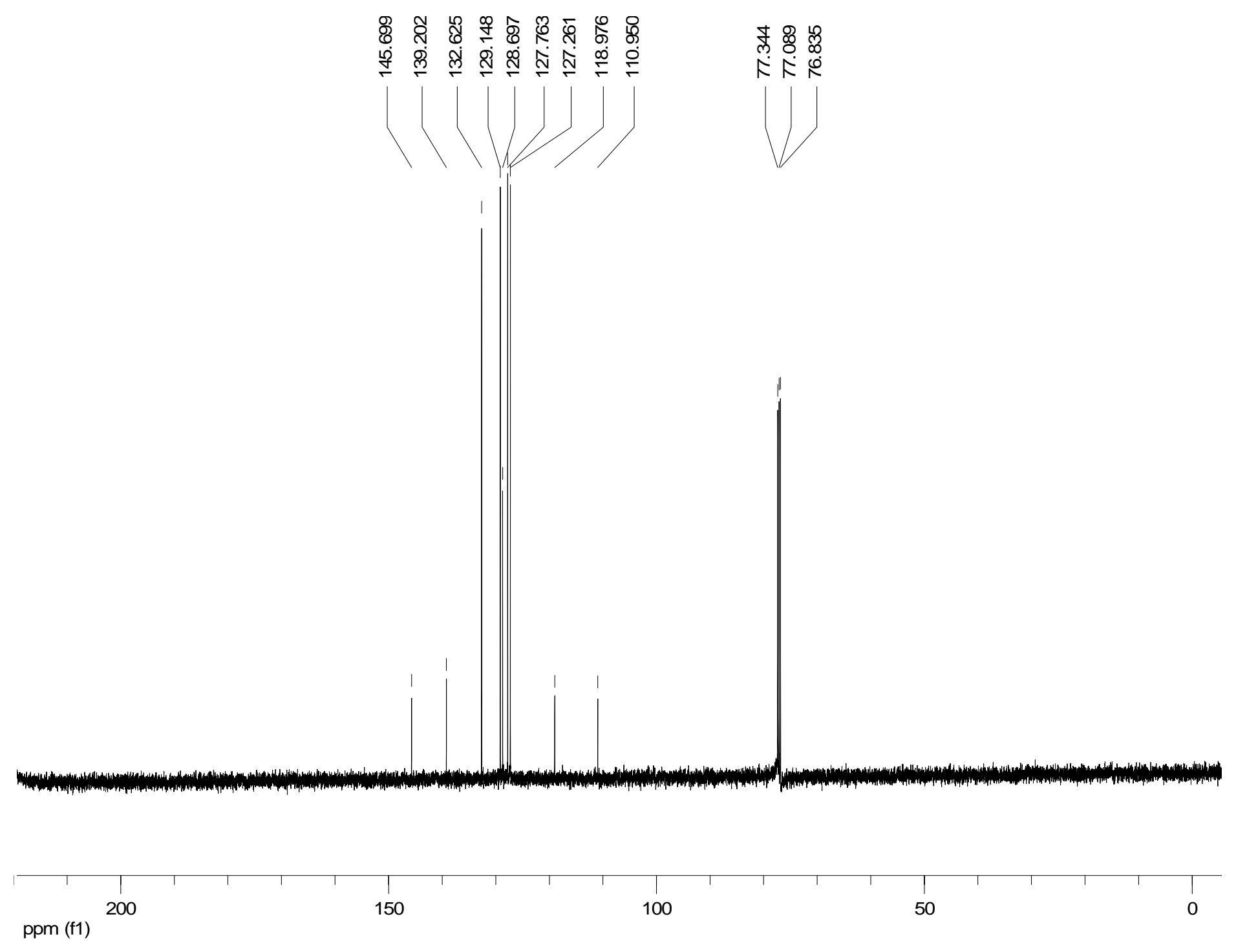


2-Methylbiphenyl

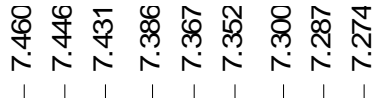

유.
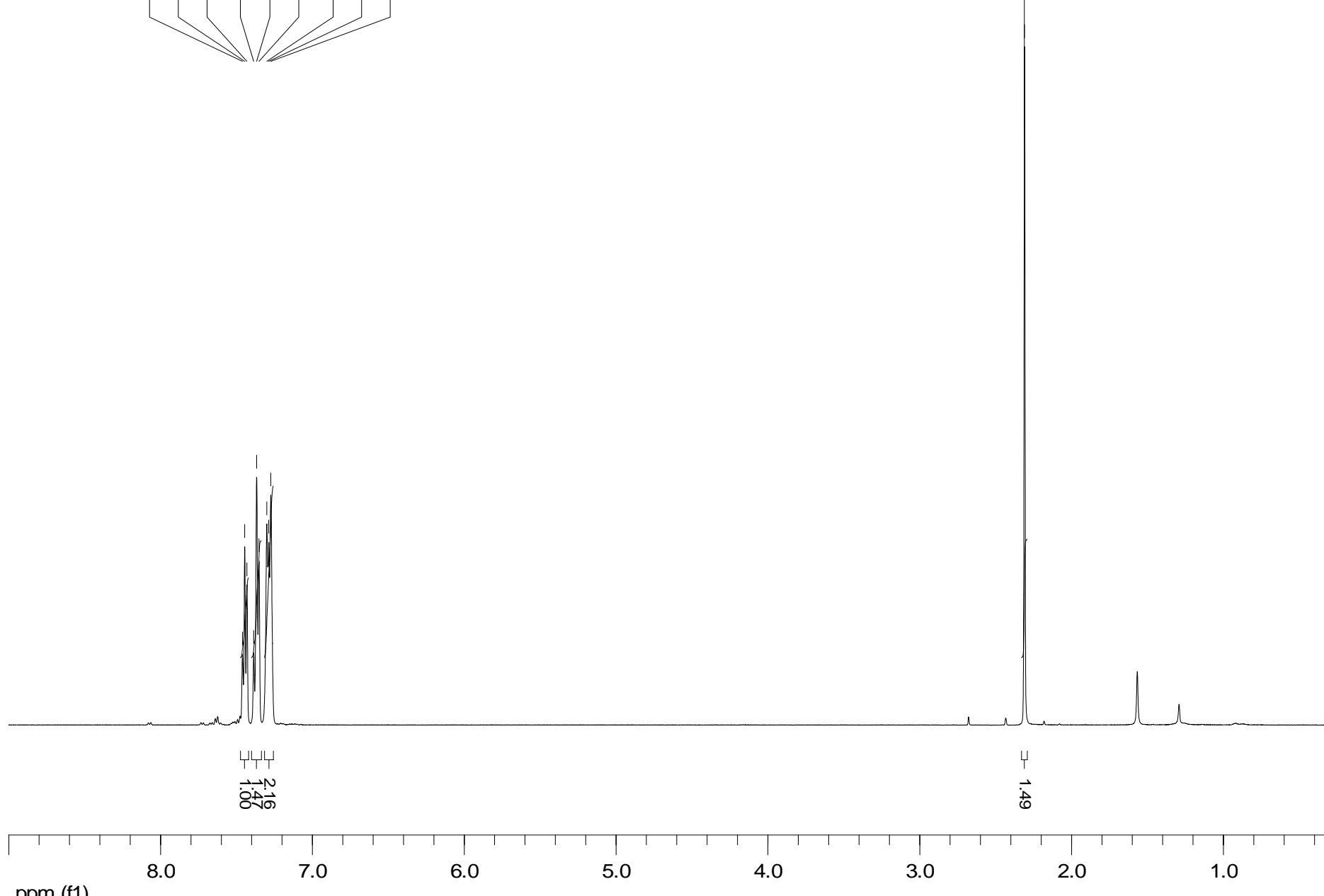

ppm (f1) 
2-Methylbiphenyl

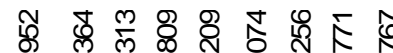

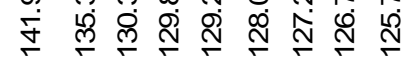

员 궁 骂点

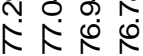

ণั่
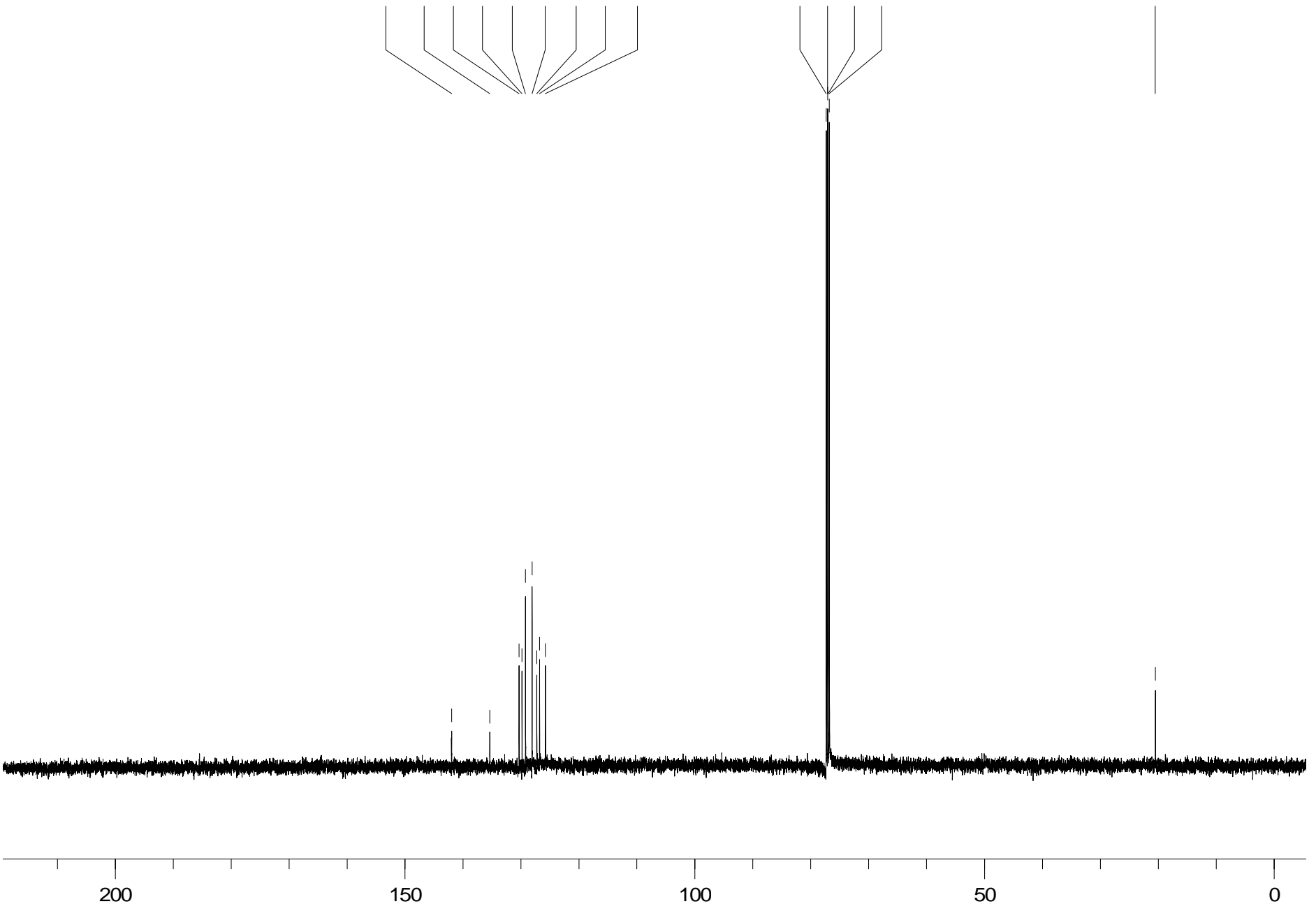

200

150

100

50

ppm (f1) 


\section{Biphenyl}

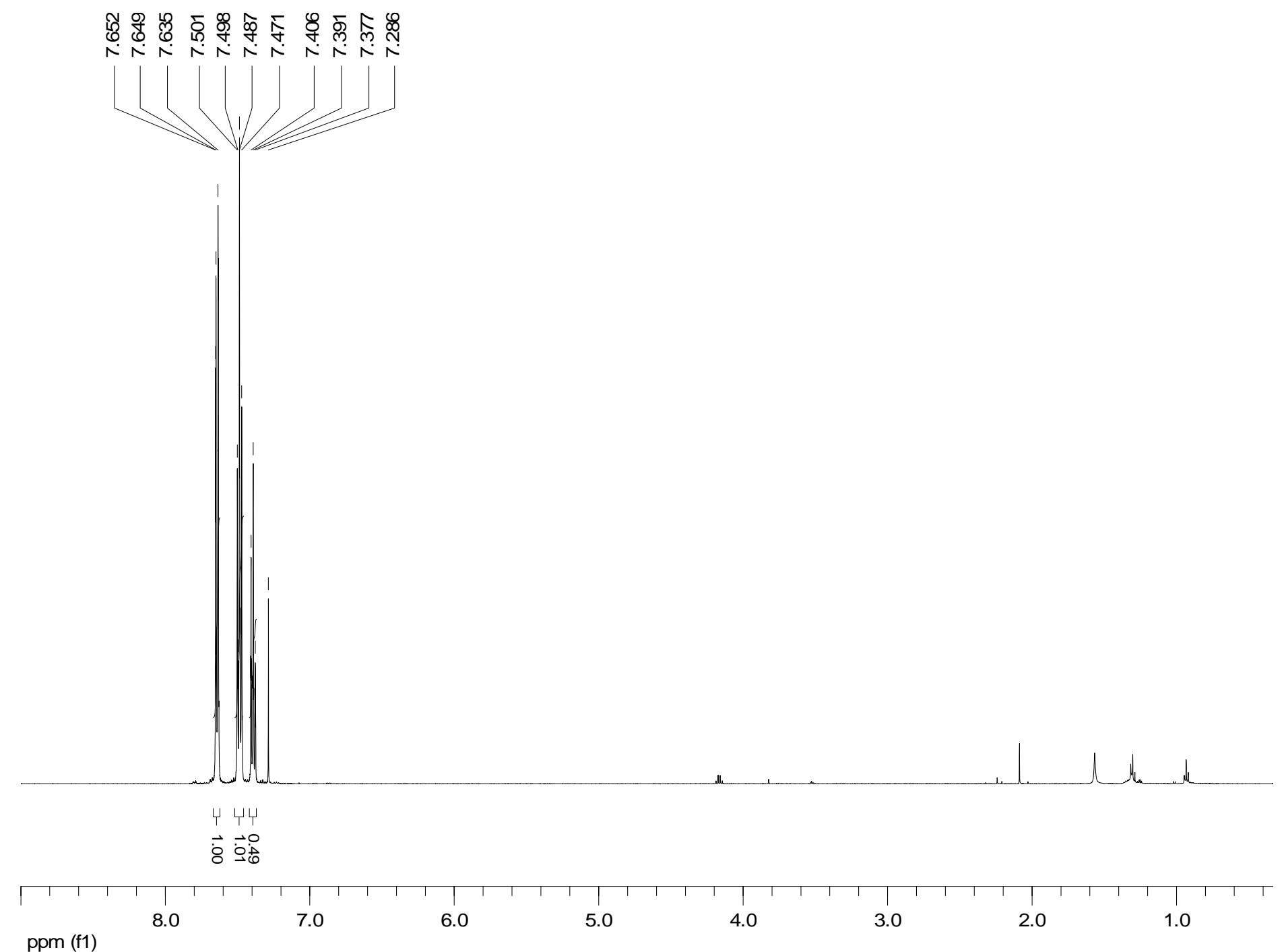


Biphenyl

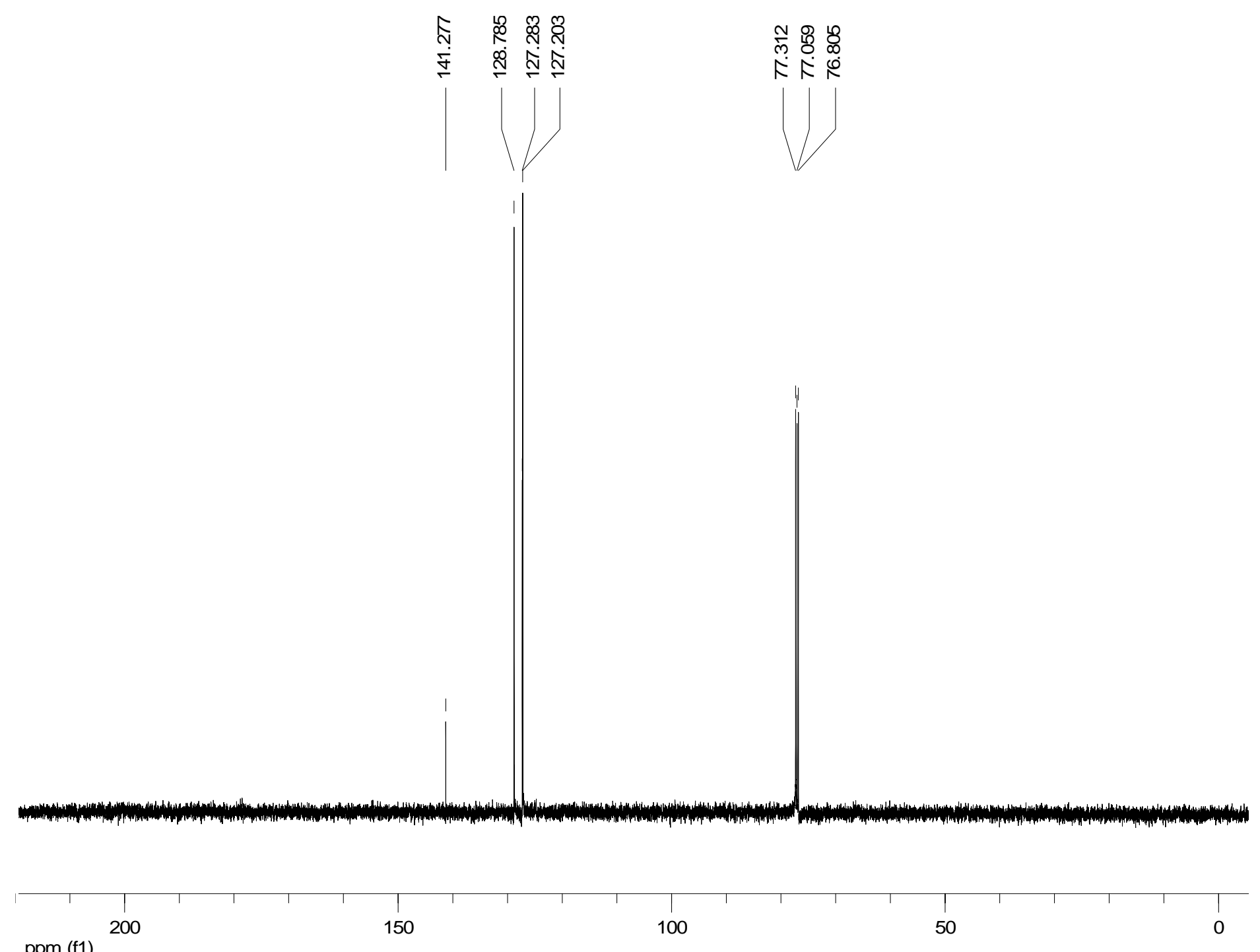

ppm (f1) 


\section{4-Methoxybiphenyl}

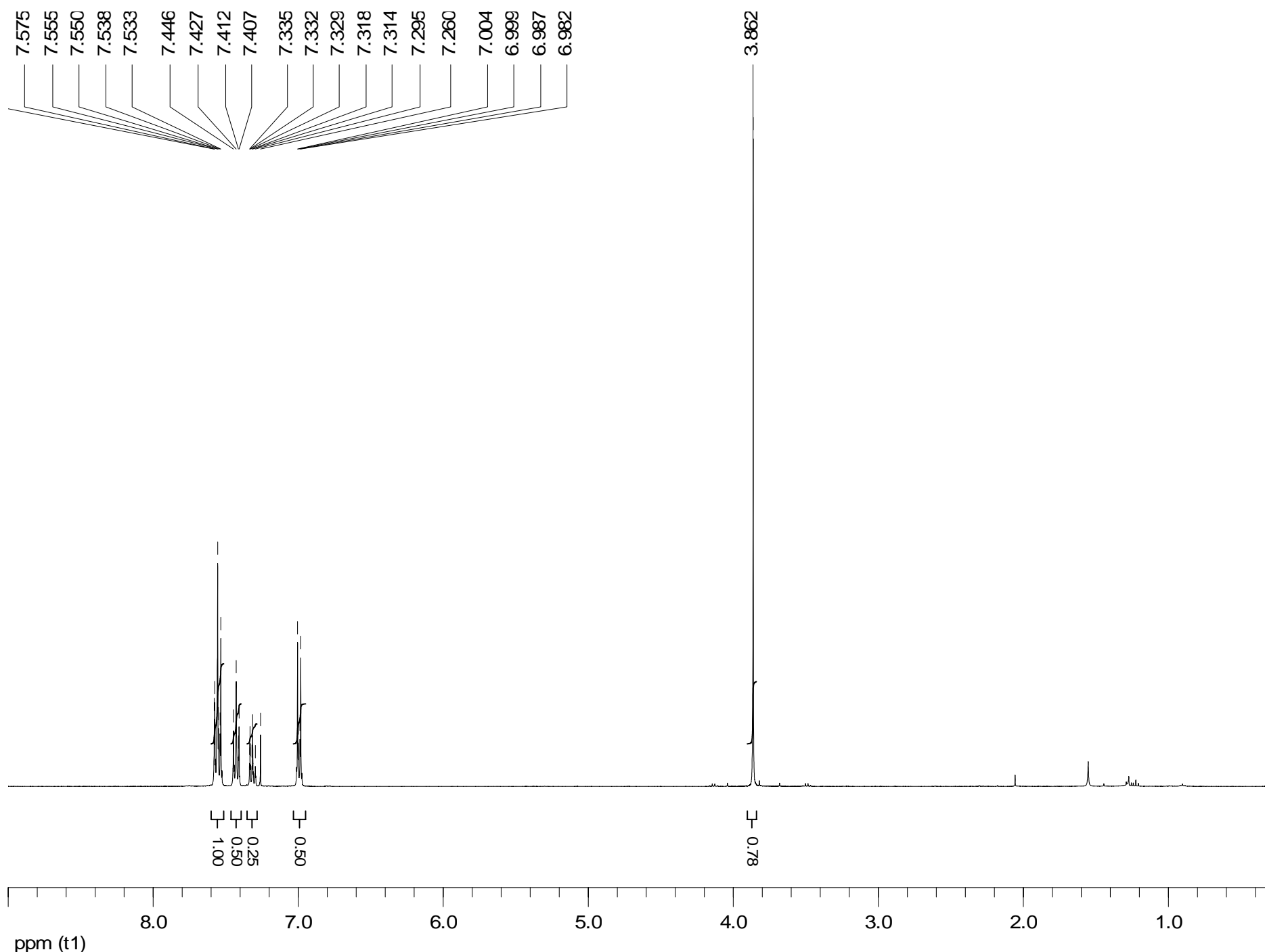


4-Methoxybiphenyl
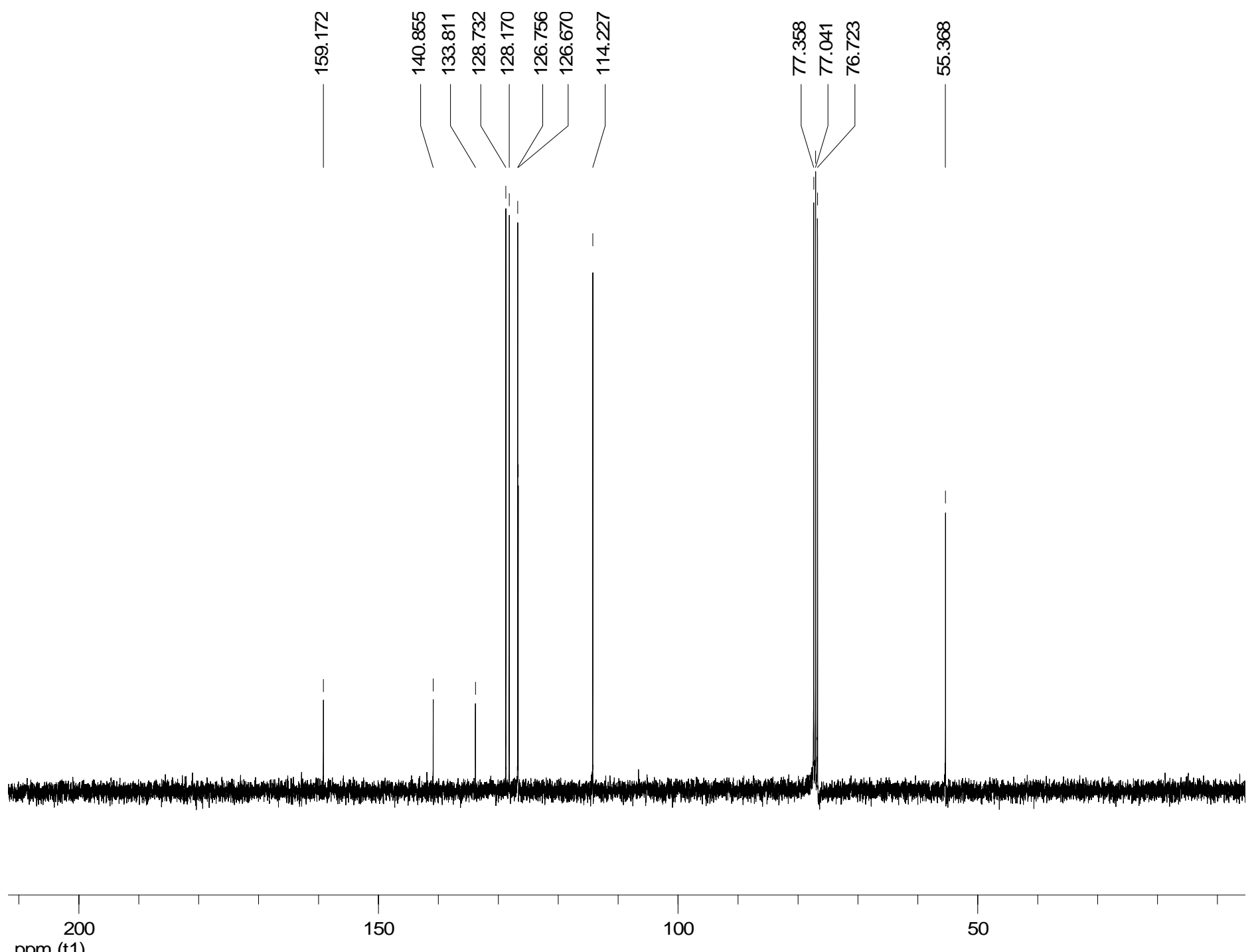

ppm (t1)

100 


\section{2-Methoxybiphenyl}

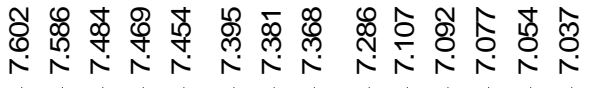
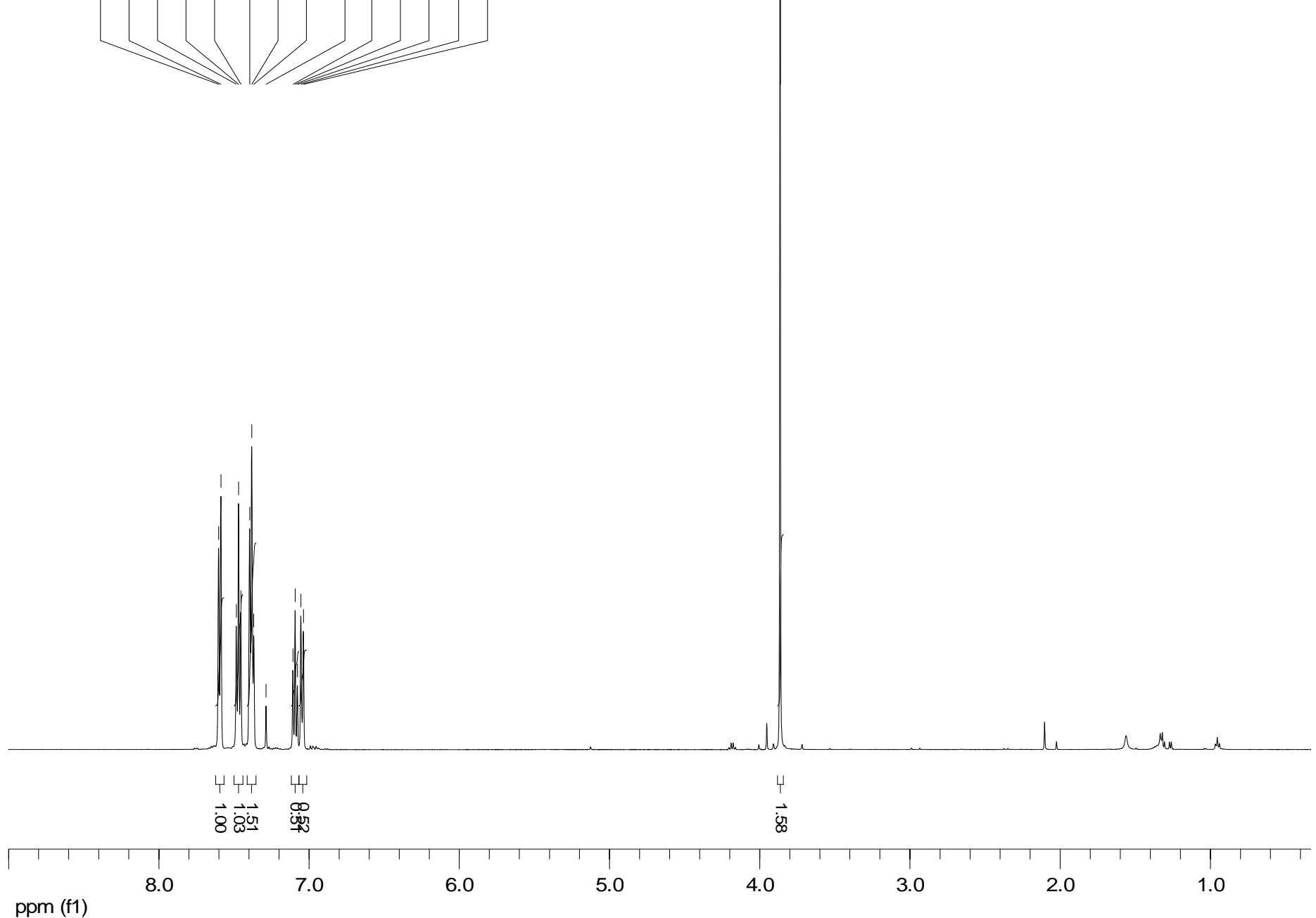
2-Methoxybiphenyl

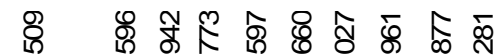

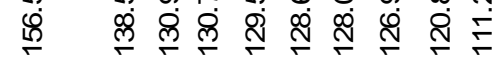

衰总总喿

ชิ

N卜

นึ่
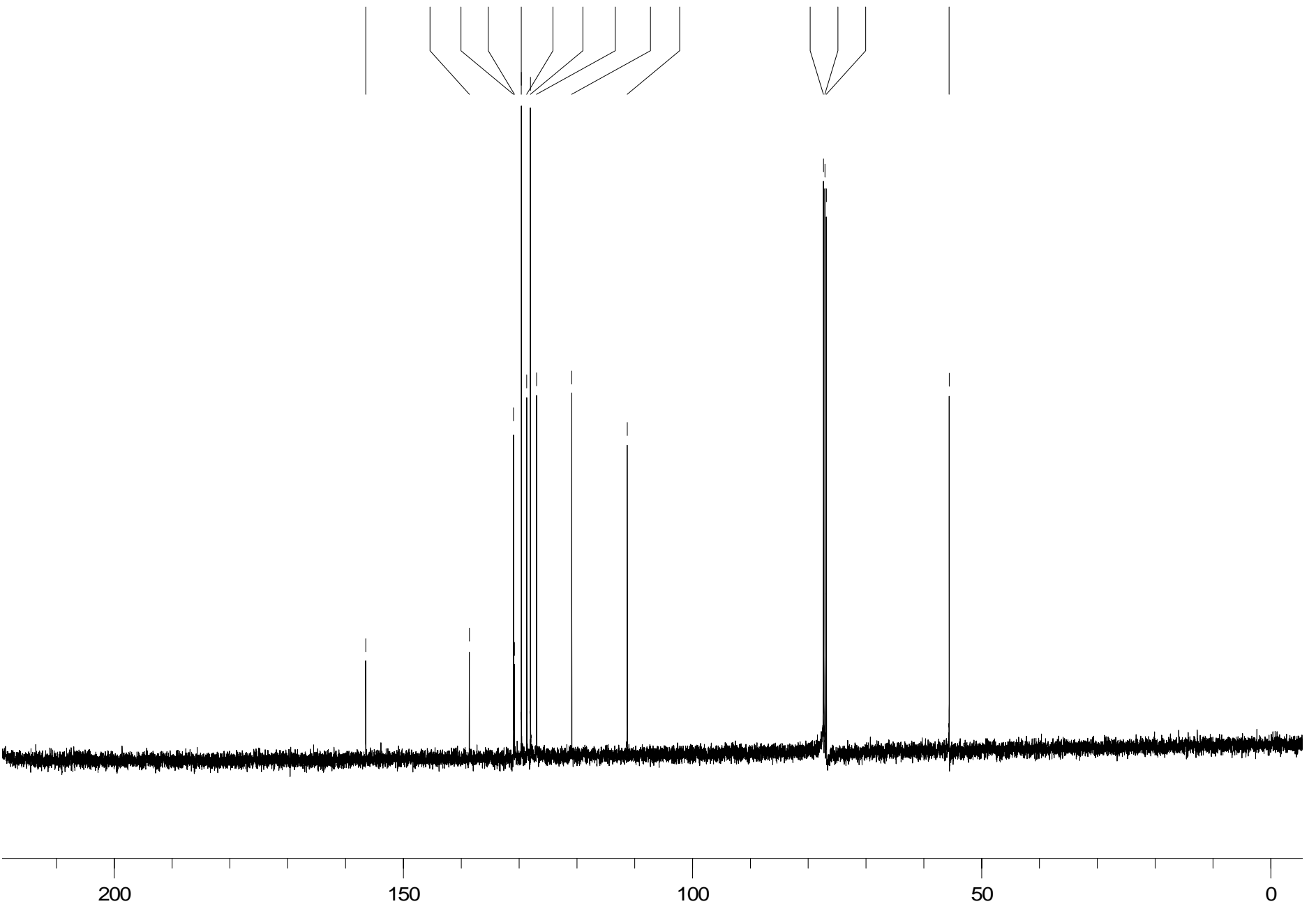

150

100

50

ppm (f1) 
4-Phenylaniline

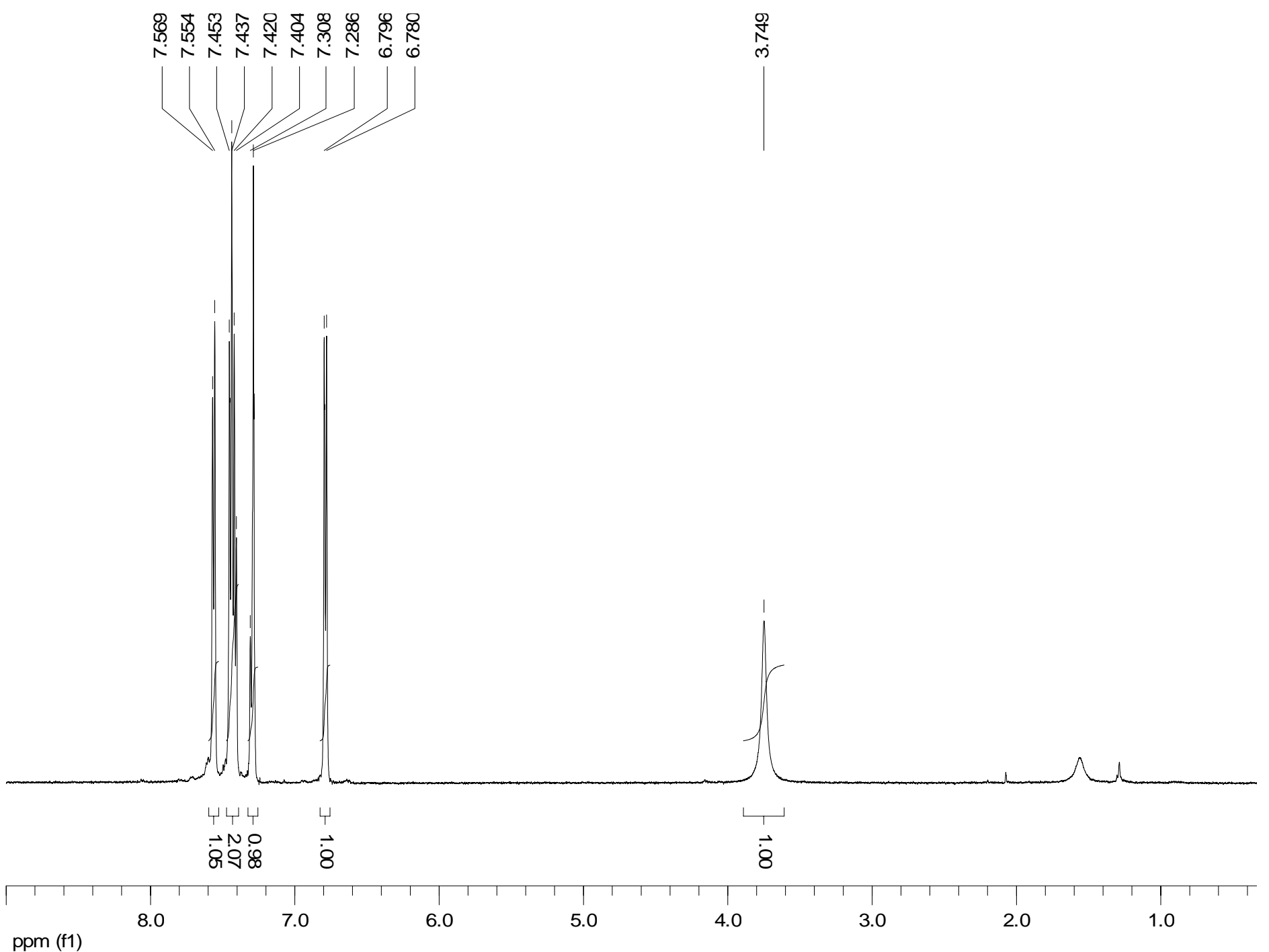


4-Phenylaniline

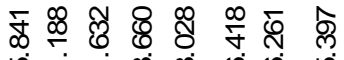

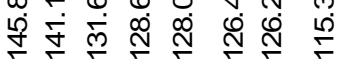

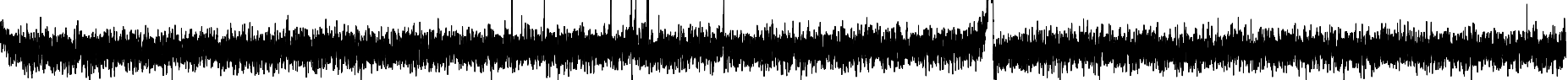

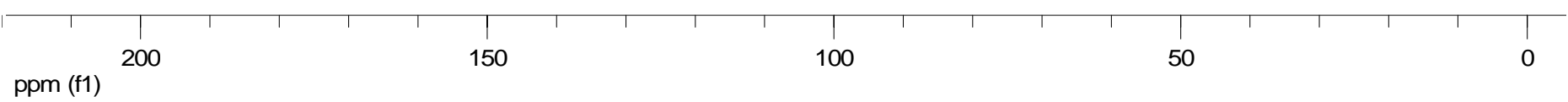


Temperature, pressure and microwave power profiles for the reaction mixture corresponding to Table 1, entry 1 (no simultaneous cooling)

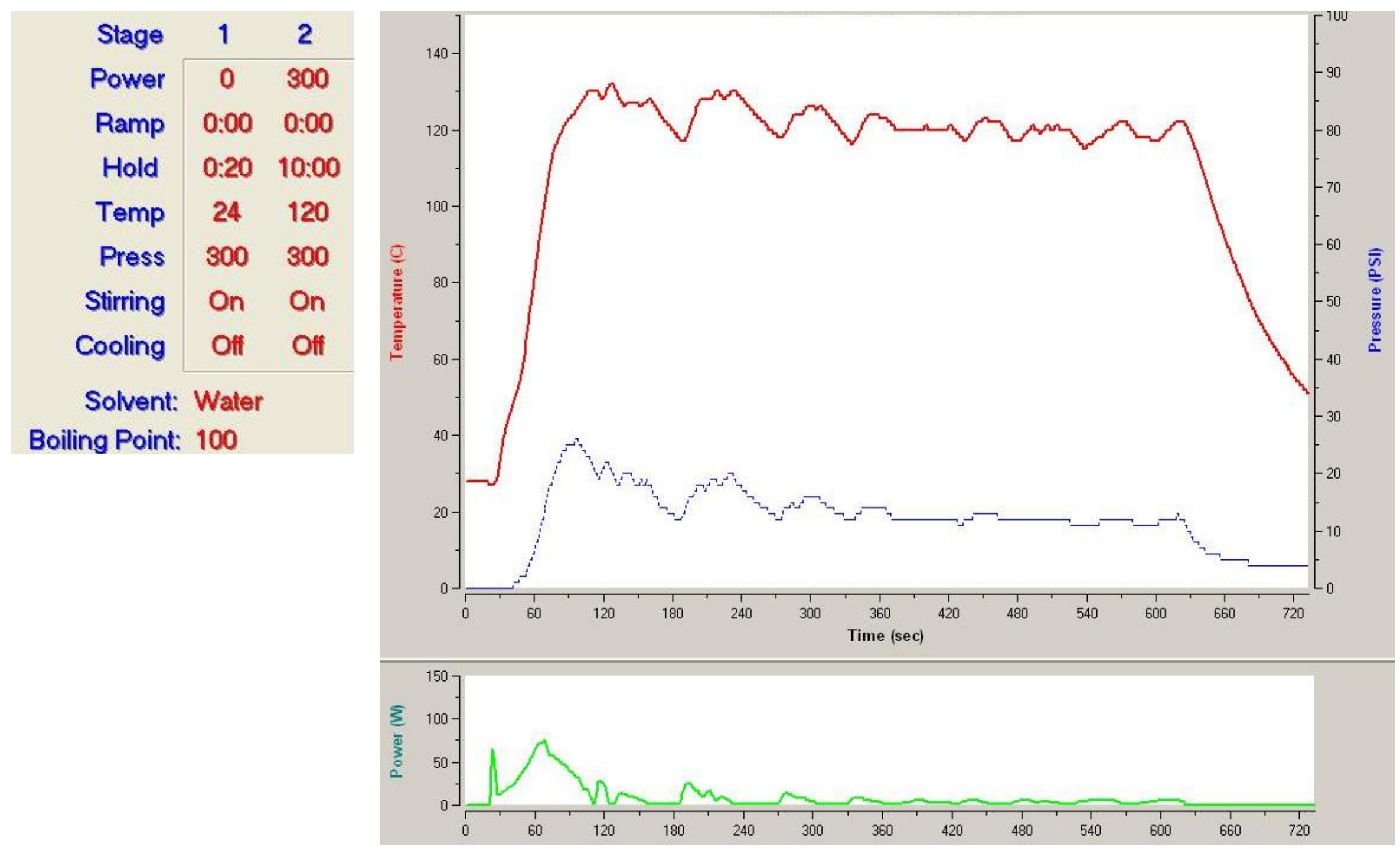


Temperature, pressure and microwave power profiles for the reaction mixture corresponding to Table 1 , entry 5 (with simultaneous cooling)

\begin{tabular}{r|cc}
\multicolumn{1}{c|}{ Stage } & 1 & 2 \\
\cline { 3 - 3 } Power & 0 & 300 \\
Ramp & $0: 00$ & $0: 00$ \\
Hold & $0: 20$ & $10: 00$ \\
Temp & 24 & 120 \\
Press & 300 & 300 \\
Stirring & On & On \\
Cooling & On & On \\
Solvent: Water & \\
Boiling Point: & 100 &
\end{tabular}
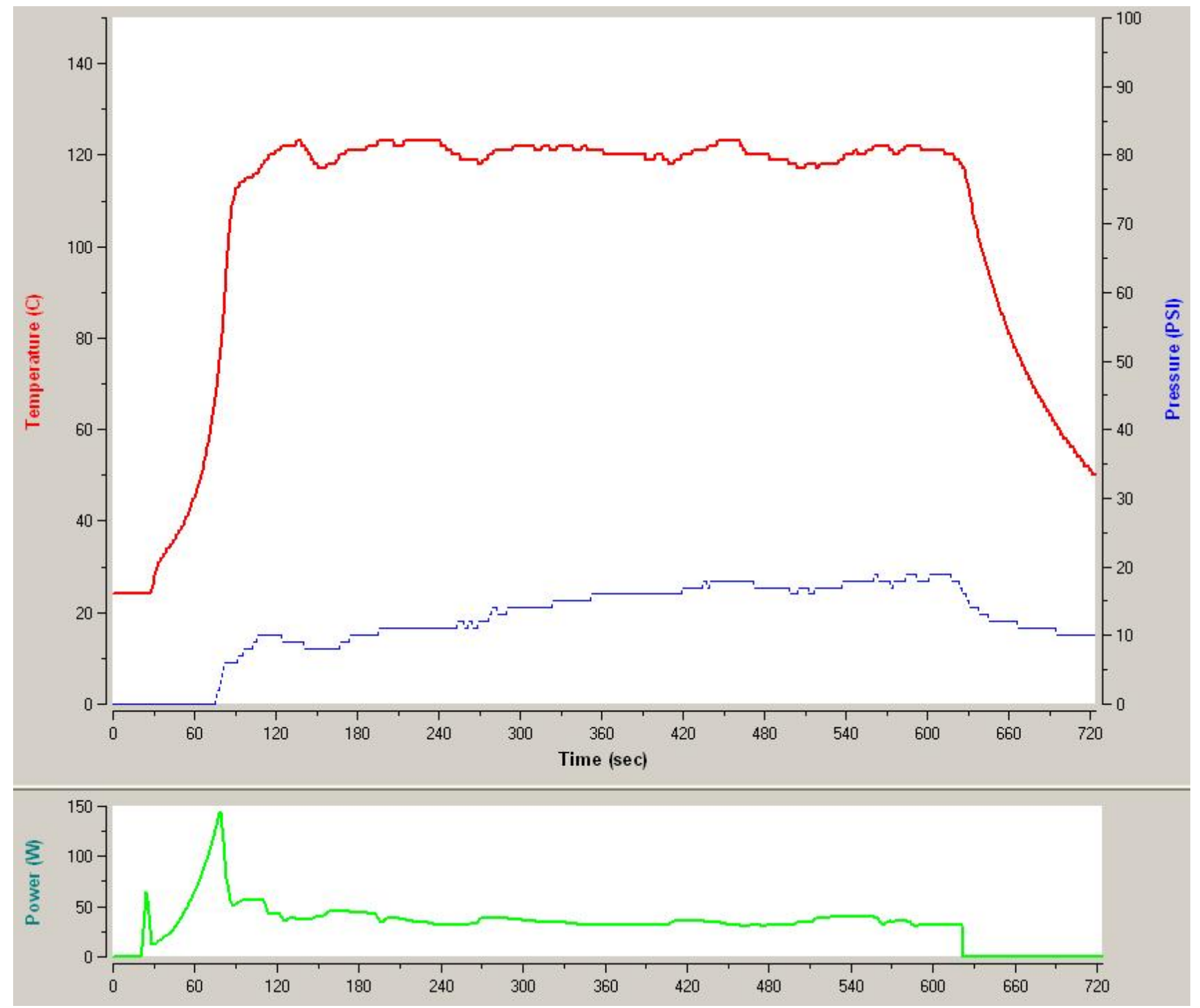